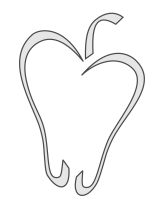

ISSN $1852-4249$

\title{
Commentary on "Experimental determination of distance and orientation of metallic nanodimers by polarization dependent plasmon coupling"
}

\author{
Sukhdev Roy ${ }^{1 *}$
}

The paper by H. E. Grecco and O. E. Martinez [1] describes an experimental technique to determine (a) sub-diffraction distances based on nearfield coupling of metallic nanoparticles, and (b) relative orientations due to symmetry breaking in the scattering cross-section. The novelty is in sequential illumination by two wavelengths to separate out the background from scattering from the nanoparticles, and in the illumination scheme to facilitate rotation of the polarization. The experimental results are shown to be in good agreement with theoretical predictions made earlier by the authors. The authors had earlier theoretically shown that the interparticle separation dependent polarization anisotropy of discrete nanoparticle dimers enables nanoscale distance measurements [2]. Their theoretical approach has also been recently experimentally implemented to simultaneously measure distance and orientation changes in discrete dimers of DNA linked nanoparticles [3].

In this Commentary, I briefly discuss some points to provide a better perspective of the contribution and make suggestions to improve the presentation. These suggestions have been taken on board by the authors and the published version of the paper is substantially improved.

Noble metal nanoparticles have been intensively

*E-mail: sukhdevroy@dei.ac.in

1 Department of Physics and Computer Science, Dayalbagh Educational Institute, Dayalbagh, Agra 282110, India studied, both theoretically and experimentally, for their unique optical properties. For a review of applications in biosystems, readers can refer for example to Ref. [4]. The excitation of the localized surface plasmon (LSP) resonance by incident electromagnetic radiation is responsible for a very large field enhancement at their surface. This singular and spatially localized optical response has proven to be of main interest for specific applications such as high sensitivity detection and spectroscopy of molecules suitably attached to the nanoparticle surface (fluorescence, Raman scattering and biochemical sensing). For a single nanoparticle, the spectral features of the LSP are known to closely depend on its size, shape and dielectric environment. More recent studies have shown that interparticle coupling effects can be used for tailoring LSP resonances with even more flexibility. This has been clearly demonstrated for nanoparticle pairs which are the most basic systems of such interacting objects.

Gold nanoparticles have stimulated tremendous research interest due to their unique optical properties. It is well established that the optical response of an individual gold nanoparticle can not only be varied by changing the dielectric environment, but more dramatically by changing the nanoparticle geometry itself. This has prompted extensive interest in the synthesis and characterization of the optical response of a wide variety of gold nanoparticle structures such as shells [5], rods [6,7], stars [8] and dumbbells [9], with the prime objective of generating a controlled plasmon resonance at a desired 
Papers in Physics, vol. 2, ART. 020011 (2010) / S. Roy

wavelength. Dimers [1-3], chains [10] and other arrays of nanoparticles are alternative methods of controlling the position or shape of the plasmon resonance. The optical properties of these arrays can be analyzed approximately in two regimes: far-field where the interparticle gap is large and the near field where the gap is sufficiently small so that the near fields of the particles are coupled. In the nearfield regime the plasmon resonance can be tuned deep into the infrared by decreasing the interparticle gap, resulting in a very strong enhancement of the electric field between the particles.

The authors address the important problem of experimental determination of distance and orientation of gold nanoparticles by measuring scattering by illumination with polarized light. They show that scattering polarization microscopy of coupled nanoparticles (with radius in the range of 4-20 nm) can provide an alternative method to fluorescence resonance energy transfer (FRET) and standard super-resolution techniques. The technique helps in filling the gap for distance and orientation measurements by these techniques, as in this range, the particles are closer than the resolution limit of the microscope and will appear as a single spot on the detector. The distance in which the technique is sensitive scales with the radii of the particles. The authors claim that the proposed technique can be an alternative to fluorescence based techniques when photostability, frame rate or coupling range are insufficient. It is interesting to note that the use of two-color imaging can provide an efficient, faster and reliable way to detect scattering centers that have plasmon resonances.

The authors should highlight the importance of the proposed contribution in the Introduction section. It should include the importance of (i) spherical metal nanoparticles and plasmon resonances, which are an indispensable tool for examining optical near-fields, imaging and sensing and (ii) distance and orientation measurements, which are very important to understand many biological systems, such as molecular structural dynamics and conformational transitions in proteins. Gold nanoparticles, in addition to their enhanced absorption and scattering, and usefulness as contrast agents in cellular and biological imaging, offer good biocompatibility, facile synthesis and conjugation to a variety of biomolecular ligands, antibodies and other targeting moieties, making them suitable for use in biochemical sensing and detection, medical diagnostics and therapeutic applications.

Since the proposed technique is based on scattering polarization microscopy, it would be in the interest of readers to also mention scanning particle enhanced Raman microscopy. Since the spectral properties of the overall plasmon resonance of two coupled spherical metal nanoparticles are subject to their material, shape, size, orientation, distance and surrounding medium, modulation of the spectral position and the spectral line width can be used to estimate the distance between two coupled metallic nanoparticles. Important related references, such as, Reinhard et al., Nano Lett. 5, 2246 (2005) and Olk et al., Nano Lett. 8, 1174 (2008) can be included to make the paper more comprehensive.

The details of the experimental setup should include parameters such as the powers or intensities of the laser beams used, the spot size of the beams at the object plane, accuracy in the angular measurements, beam splitting ratio, bandwidth of the filters used, etc. to facilitate better understanding of the experiment. It should be clarified whether black and white or color recordings were made. A color CCD camera can improve the identification of particles by efficient measurement of scattered intensities and their color. A discussion on the photostability of the proposed technique, which has been mentioned as the main advantage over fluorescence and super-resolution based techniques in the Results and Discussion section, would provide justification and highlight the importance of scattering polarization microscopy.

Since it is an experimental study, a discussion on the various factors that can lead to uncertainty in the distance and orientation measurement, such as non-uniformity of the particles (both size and shape), noise, accuracy of the measurement of angles etc., is required to clearly assess the limitations and help in future efforts to improve the technique. Since relative error depends on distance, it would be good to mention where the best spatial resolution for inter-particle separation occurs for $20 \mathrm{~nm}$ Au plasmon ruler.

The efficient use of two color imaging to detect scattering centers demonstrated in the illumination setup by Grecco and Martínez in combination with other techniques, provides a robust anisotropy based microscopic tool for further studies. 
[1] H E Grecco, O E Martínez, Experimental determination of distance and orientation of metallic nanodimers by polarization dependent plasmon coupling, Pap. Phys. 2, 020010 (2010).

[2] H E Grecco, O E Martínez, Distance and orientation measurement in the nanometric scale based on polarization anisotropy of metallic dimers, Opt. Exp. 14, 8716 (2006).

[3] H Wang, B M Reinhard, Monitoring simultaneous distance and orientation changes in discrete dimers of DNA linked gold nanoparticles, J. Phys. Chem. C 113, 11215 (2009).

[4] P K Jain, X Huang, I H El-Sayed, M A ElSayed, Review of some interesting surface plasmon resonance-enhanced properties of noble metal nanoparticles and their applications to biosystems, Plasmonics 2, 107 (2007).

[5] S J Oldenburg, R D Averitt, S L Westcott, N J Halas, Nanoengineering of optical resonances, Chem. Phys. Lett. 288, 243 (1998).
[6] C J Murphy, T K Sau, A M Gole, C J Orendorff, J Gao, L Gou, S E Hunyadi, T Li, Anisotropic metal nanoparticles: Synthesis, assembly, and optical applications, J. Phys. Chem. B 109, 13857 (2005).

[7] L S Slaughter, Y Wu, B A Willingham, P Nordlander, S Link, Effects of asymmetry breaking and conductive contact on the plasmon coupling in gold nanorod dimers, ACS Nano 4, 4657 (2010).

[8] C L Nehl, H Liao, J H Hafner, Optical properties of star-shaped gold nanoparticles, Nano Lett. 6, 683 (2006).

[9] D K Lim, K S Jeon, H M Kim, J M Nam, Y D Suh, Nanogap-engineerable Raman-active nanodumbbells for single-molecule detection, Nat. Mater. 9, 60 (2010).

[10] N Harris, M D Arnold, M G Blaber, M J Ford, Plasmonic resonances of closely coupled gold nanosphere chains, J. Phys. Chem. C 113, 2784 (2009). 